\title{
Le récepteur de l'insuline, second messager de l'hormone
}

L'utilisation des méthodes des recombinants d'ADN a permis de progresser rapidement dans l'étude de la structure et de la fonction du récepteur de l'insuline. Ses deux chaînes $\alpha$ et $\beta$ dérivent d'un précurseur commun unique. La chaîne $\alpha$ est exclusivement extracellulaire et contient le domaine de fixation de l'hormone. La chaîne $\beta$ comporte une région extracellulaire et possède un segment transmembranaire ainsi qu'une importante région intracytoplasmique, support d'une activité de tyrosine kinase. Cette activité, stimulée par la liaison de l'hormone au récepteur, est indispensable à la transmission à la cellule du message métabolique de l'insuline. Des anomalies génétiques du récepteur sont activement recherchées dans une série d'affections comportant une résistance à l'insuline.

\section{Éric Clauser}

Chargé de recherche au Cnrs

ADRESSE

E. Clauser: Inserm U. 36, 17, rue du Fer à

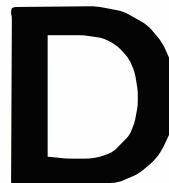

epuis la découverte de l'insuline par Banting et Best en 1922 et de l'implication de cette hormone dans la genèse du diabète sucré, un intérêt considérable s'est développé pour ce polypeptide et son mode d'action. Sa sequence primaire et sa structure tridimensionnelle ont été déterminées, ses actions physiologiques analysées au niveau de chacun des tissus cibles (foie, muscle, tissu adipeux, en particulier). Parallèlement à ces travaux, certains facteurs de croissance peptidiques, comme les facteurs de croissance I et II proches de l'insuline (IGFl ou somatomédine C et IGF2) étaient découverts ; ils ont avec l'insuline d'importantes similitudes de séquence $(50 \%)$ associées à certains effets physiologiques communs. En revanche, le facteur de croissance épidermique (EGF), chef de file des facteurs de croissance, a une structure très différente de celle de l'insuline.

L'obtention d'insuline pure et la mise au point de son marquage radioactif ont permis la mise en évidence de sites de fixation membranaires. Une investigation détaillée de la synthèse et du métabolisme de ce récepteur dans les différents tissus était entreprise, alors qu'une première image, encore imprécise, de sa structure, se dessinait. Mais ce n'est qu'en 1980 que sont apparues les premières preuves expérimentales montrant que ce recepteur était non seulement une unité de liaison, mais pouvait aussi jouer un rôle actif dans la «transduction» du signal vers l'intérieur de la cellule. Les démonstrations successives qu'à l'unité de liaison de l'hormone était couplée une enzyme, que cette activité enzymatique était de type tyrosine kinase et qu'elle était portée par la molécule de récepteur pour l'insuline [1] ont conduit au concept de récepteurenzyme, valable aussi pour de nombreux autres récepteurs tels ceux de l'EGF et de l'IGFl.

Le développement récent des techniques de recombinants d'ADN a permis, par clonage 
et séquençage nucléotidique des ADN complémentaires respectifs, de déduire l'enchaînement primaire des acides aminés du récepteur de l'insuline $[2,3]$, de l'EGF [4] et de l'IGFl [5]. Ceci fournissait les informations et le matériel nécessaire à une étude précise et détaillée des rapports entre la structure et les fonctions de cette nouvelle classe de récepteurs, ainsi que des possibilités d'étudier les mécanismes de transmission, à l'intérieur de la cellule, du message porté par l'hormone peptidique strictement extracellulaire. Les progrès récents dans la connaissance de la structure, du fonctionnement et des éventuelles anomalies du récepteur de l'insuline seront détaillés successivement dans cet article.

\section{Structure moléculaire}

Le clonage de l'ADN complémentaire du messager codant pour le récepteur de l'insuline a été réalisé simultanément par deux équipes californiennes $[2,3]$. Leur stratégie, à peu près identique, a consisté à purifier le récepteur à partir du placenta par chromatographies d'affinité successives sur une colonne d'agglutinines de germe de blé, puis sur une colonne d'anticorps spécifiques. Les deux chaînes du récepteur ont ensuite été séparées par électrophorèse sur gel de polyacrylamide et microséquencées à leur extrémité $\mathrm{NH}_{2}$ terminale. Des séquences internes de la chaîne $\alpha$ ont également été déterminées après digestion trypsique. De la séquence $\mathrm{NH}_{2}$ terminale de la chaîne $\alpha$ a pu être déduite une séquence oligonucléotidique, non univoque du fait de la dégénérescence du code génétique. C'est à partir de cette séquence qu'ont été synthétisés les oligonucléotides utilisés pour le criblage des banques d'ADN complémentaires. Il s'agissait d'un mélange de 268 oligonucléotides homologues de 32 résidus de long (32 mers) dans un cas et d'un 63 mer unique dans le deuxième cas. Les deux équipes ont utilisé des banques d'ADNc synthétisés à $\mathrm{m} / \mathrm{s} n^{\circ} 2$ vol. 4 , feurier 88 partir d'ARN messager de placenta humain, sélectionnés ou non selon leur taille et clonés dans les phages lambda gt $10 \mathrm{ou}$ 11 utilisés comme vecteurs.

Des clones, contenant des insertions de 4 à $5 \mathrm{~kb}$ (kilobases), ont été isolés grâce aux sondes oligonucléotidiques marquées. Les insertions ont été séquencées, permettant de déduire la séquence en acides aminés des deux chaînes du récepteur de l'insuline. Il faut souligner que les deux séquences, déterminées de façon indépendante, étaient identiques, à l'exception d'une insertion de 12 acides aminés à l'extrémité COOH terminale de la chaîne $\alpha$ non retrouvée par l'une des équipes. L'existence de deux types de récepteurs a été confirmée depuis dans différents tissus sans qu'il soit possible d'établir à ce jour une différence de fonction entre ces molécules.

Une séquence de plus de 4000 bases code pour les 1355 acides aminés du récepteur de l'insuline. Cette séquence débute par le peptide signal, nécessaire au routage des protéines membranaires et sécrétoires et représenté ici par une séquence de 27 acides aminés essentiellement hydrophobes. La chaîne $\alpha$ (figure 1, page 74) du récepteur suit immédiatement le peptide signal et représente un enchaînement de 735 acides aminés. L'absence de séquence hydrophobe fait de cette chaîne une protéine essentiellement extracellulaire. L'attachement de cette sous-unité à la membrane cellulaire et à la sous-unité $\beta$ se fait très probablement par l'intermédiaire de ponts disulfures. Les cystéines de la chaîne $\alpha$ sont d'ailleurs concentrées dans une région de 157 acides aminés à la jonction du premier tiers et des deux autres tiers de la sous-unité. Cette région, dite « riche en cystéines », contient en effet $70 \%$ de résidus hydrophiles et 26 des 37 cystéines extracellulaires. La conservation quasi parfaite de la position de ces résidus cystéines dans le domaine homologue des récepteurs de l'IGFl ( $50 \%$ d'identité) et de l'EGF (20\%) font de cette région un domaine fonctionnel conservé. De telles régions hydrophiles et riches en cystéines ont été mises en évidence pour des récepteurs aussi différents que le récepteur des lipoprotéines de basse densité (LDL), voire même les récepteurs des hormones stéroïdes. Dans ce dernier cas, cependant, la structure et la fonction de ce domaine sont bien différentes puisque ce dernier est intracellulaire et se lie aux séquences régulatrices spécifiques d'ADN. En l'absence d'information précise sur ce que doit être le site de liaison pour l'insuline et bien que le site de liaison de la molécule d'insuline soit connu, il n'est pas possible à ce jour d'identifier les acides aminés de la chaîne $\alpha$ impliqués dans la liaison de l'hormone. Le clivage entre les sous-unités $\alpha$ et $\beta$ est effectué au niveau de la séquence arg-lys-argarg (732-735) très probablement par une enzyme de la famille de la trypsine.

La chaîne $\beta$ comporte un court domaine extracellulaire (180 acides aminés) contenant quatre résidus cystéines. Il précède le domaine transmembranaire fait de 23 acides aminés hydrophobes, puis trois acides aminés basiques. La portion intracellulaire de la chaîne $\beta$ est composée d'un domaine tyrosine kinase identifiable, entre autre, par son site de liaison de l'ATP (gly-gln-gly-ser-phe-gly... lys [1003-1020]). Les homologies de ce domaine avec d'autres tyrosine kinases sont tout à fait remarquables (figure 2, page 75); elles sont observées non seulement avec les domaines équivalents du récepteur de l'IGFl $(80 \%)$, de l'EGF (30\%) et des récepteurs d'autres facteurs de croissance tel que PDGF, mais aussi avec les tyrosine kinases codées par les oncogènes tels que $v$-ros (49\%) et oncD $(43 \%)$. La comparaison de ces séquences montre que parmi les 13 résidus tyrosyl de la chaîne $\beta$ du récepteur de l'insuline, seules les tyrosines jumelles 1162-1163 sont particulièrement conservées dans la famille 


\section{RÉFÉRENCES}

1. Kasuga M, Zick Y, Blith DL, Karlssom FA, Karing HU, Kahn CR. Insulin stimulation of phosphorylation of the $\beta$-subunit of the insulin receptor. Formation of both phosphoserine and phosphotyrosine. J Biol Chem 1982 ; 257 : $9891-4$.

2. Ebina $\mathrm{Y}$, Ellis $\mathrm{L}$, Jarnagin $\mathrm{K}$, et al. The human insulin receptor CDNA : The structural basis for hormone-activated transmembrane signalling. Cell 1985; 40 : 747-58.

3. Ullrich A, Bell JR, Chen EY, et al. Human insulin receptor and its relationships to the tyrosine kinase family of oncogenes. Nature 1985 ; 313 : 756-61

4. Ullrich A, Coussens L, Hayflick JS, et al. Human epidermal growth factor receptor cDNA sequence and aberrant expression of the amplified gene in A431 epidermoid carcinoma cells. Nature 1984 ; 309: 418-25.

5. Ullrich A, Gray A, Tam AW, et al. Insulinlike growth factor I receptor primary struc. ture : comparison with insulin suggests structural deteminants that define functional specificity. $E M B O$ J 1986; 5 : 2503-12.

6. Morgan DO, Edman JC, Standring DN, et al. Insulin-like growth factor II receptor as a multifunctional binding protein. Nature 1987 ; 329 : $301-7$.

7. Herzberg VL, Grigorescu F, Edge ASB, Spiro RG, Kahn CR. Characterization of insulin receptor carbohydrate by comparison of chemical and enzymatic glycosylation. Biochem Biophys Res Commun 1985; 129 : 789. 96.

8. Kahn CR, Baird KL, Jarett DB, Flier JS. Direct demonstration that receptor cross-linking aggregation is important in insulin action. Proc Natl Acad Sci USA 1978; 75 : 4209-13.

9. Fujita-Yamaguchi Y, Kathuria S. The monomeric $\alpha \beta$ form of the insulin receptor exhibits much higher insulin-dependent tyrosine-specific protein kinase activity than the intact $\alpha_{2} \beta_{2}$ form of the receptor. Proc Natl Acad Sci USA 1985 ; 82 : 6095-9.

10. Meyts $P$ (de), Van Obberghen $E$, Roth J, Wollmer A, Brandenbourg D. Mapping of the residues responsible for the negative cooperativity of the receptor binding region of insu- des tyrosine kinases, où elles sont généralement un site préférentiel d'autophosphorylation. Cette région de 250 acides aminés environ est suivie d'une région $C$ terminale de 100 acides aminés. Ce dernier domaine, essentiellement hydrophile, est spécifique du récepteur de l'insuline. Malgré les similitudes entre des récepteurs pour des ligands homologues, il faut cependant souligner que le récepteur de l'IGF2 paraît faire exception, puisqu'il s'agit d'une glycoproteine monocaténaire d'un poids moléculaire de 250000 ne possédant pas d'activité tyrosine kinase. Le clonage récent de

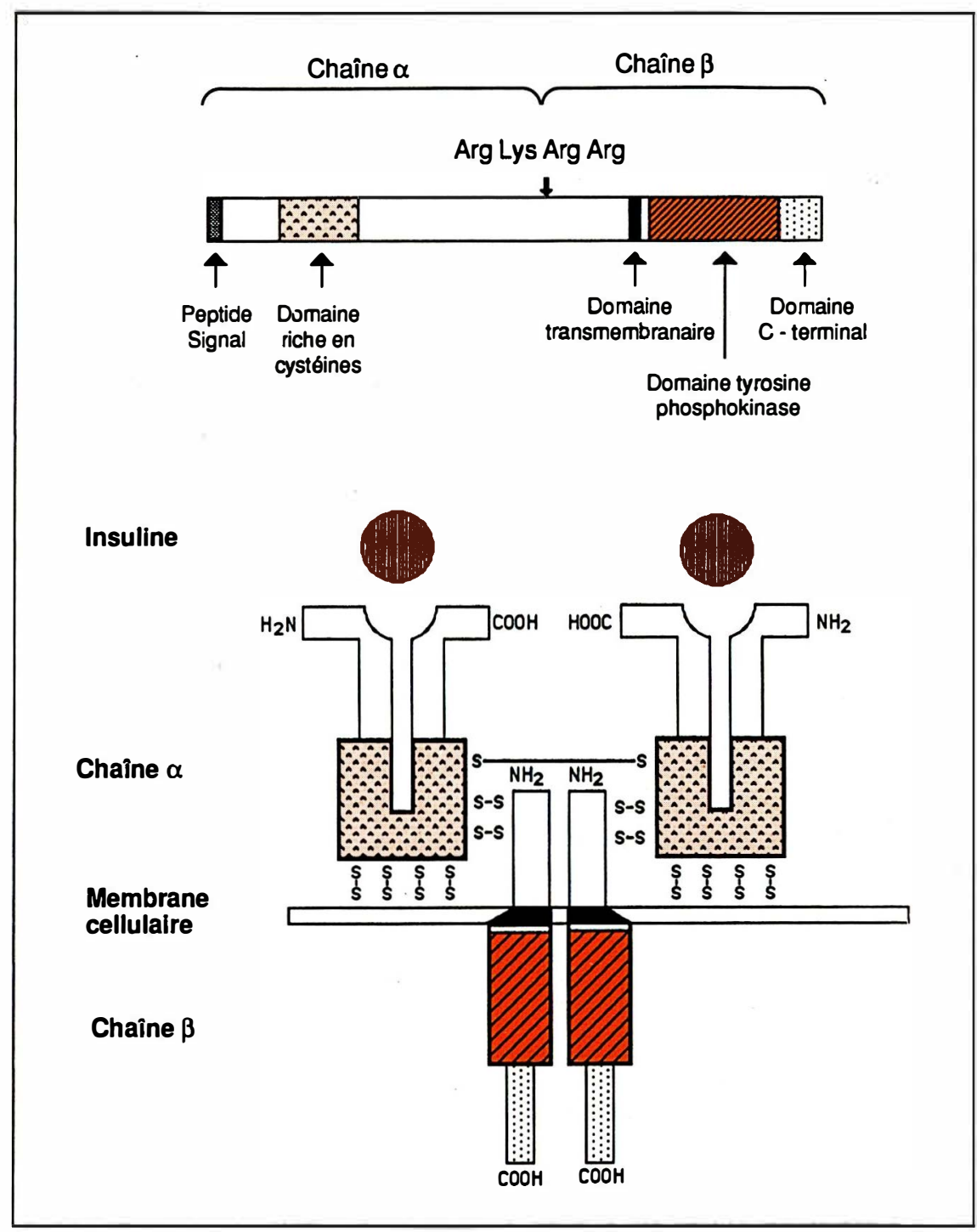

Figure 1. Représentations schómatiques do la structuro du récopteur do l'insulino. En haut, sont représentés les différents domaines fonctionnels des deux chaînes de la protéine, tels qu'ils sont codés par I'ARN messager. Le site de coupure protéolytique séparant les deux chaînes par clivage du précurseur est indiqué (arg-lys-arg-arg). En bas, la protéine est schématisée, en tenant compte des connaissances ou des hypothèses les plus probables concernant la fonction des différents domaines. Ainsi la chaîne $\alpha$, entièrement extracellulaire, comporte des séquences impliquées dans la liaison de l'insuline. Son domaine riche en cystéines assure la rigidité et l'amarrage membranaire de la chaîne. La chaîne $\boldsymbol{\beta}$ comporte un domaine extracellulaire, un domaine transmembranaire, le domaine tyrosine kinase intracellulaire et la région $C$ terminale libre dans le cytoplasme. La structure fonctionnelle pourrait se présenter sous la forme d'un hétérodimère $\alpha_{2} \beta_{2}$. 
son ADNc [6] confirme ces données et montre qu'il s'agit d'une protéine identique au récepteur indépendant des cations des résidus mannose 6 phosphate associé aux enzymes lysosomales et composé de 15 répétitions d'un domaine de base. Seule une courte séquence de six acides aminés incluant une tyrosine, située immédiatement après le domaine transmembranaire du récepteur de l'IGF2, est conservée en position homologue pour tous les membres de la famille des récepteur-kinases. La signification de cette séquence est inconnue.

Les chaînes $\alpha$ et $\beta$ ont respectivement 13 et 4 sites potentiels de $\mathbf{N}$ glycosylation. Les sucres rendent compte en effet de la différence existant entre le poids moléculaire déterminé $(220000)$ et celui calculé. Cette différence représente environ $30 \%$ du poids du récepteur. La nature de ces sucres n'est pas parfaitement connue mais ils comprennent à la fois des sucres riches en mannose ou des oligosaccharides complexes liés aux asparagines ( $\mathrm{N}$-glycosylation) et des sucres liés aux sérines et thréonines principalement sur la chaîne $\beta$ (O-glycosylation) [7]. De nombreux auteurs s'accordent pour penser que l'unité fonctionnelle du récepteur est un hétérotétramère $\alpha_{2} \beta_{2}$, plutôt qu'un hétérodimère $\alpha \beta$. Cette hypothèse repose sur deux types d'arguments : (1) sur le plan biochimique le récepteur purifié se présente sous une forme essentiellement $\alpha_{2} \beta_{2}$ en conditions non dénaturantes; (2) certains anticorps anti-récepteurs entiers ont un effet insulino-mimétique in vivo, alors que leurs fragments Fab sont inefficaces; cet effet est restauré par l'adjonction d'immunoglobulines anti-Fab [8]. Cette expérience a été interprétée de la manière suivante: ces anticorps anti-récepteurs seraient insulinomimétiques, non pas en occupant de façon spécifique le site de liaison de l'insuline, mais en se liant à un autre épitope des chaînes $\alpha$. La liaison à deux chaînes $\alpha(\operatorname{IgG})$ et non à une seule (Fab) provoquerait des modifications de $m / s n^{\circ} 2$ vol. 4, fámiar 88

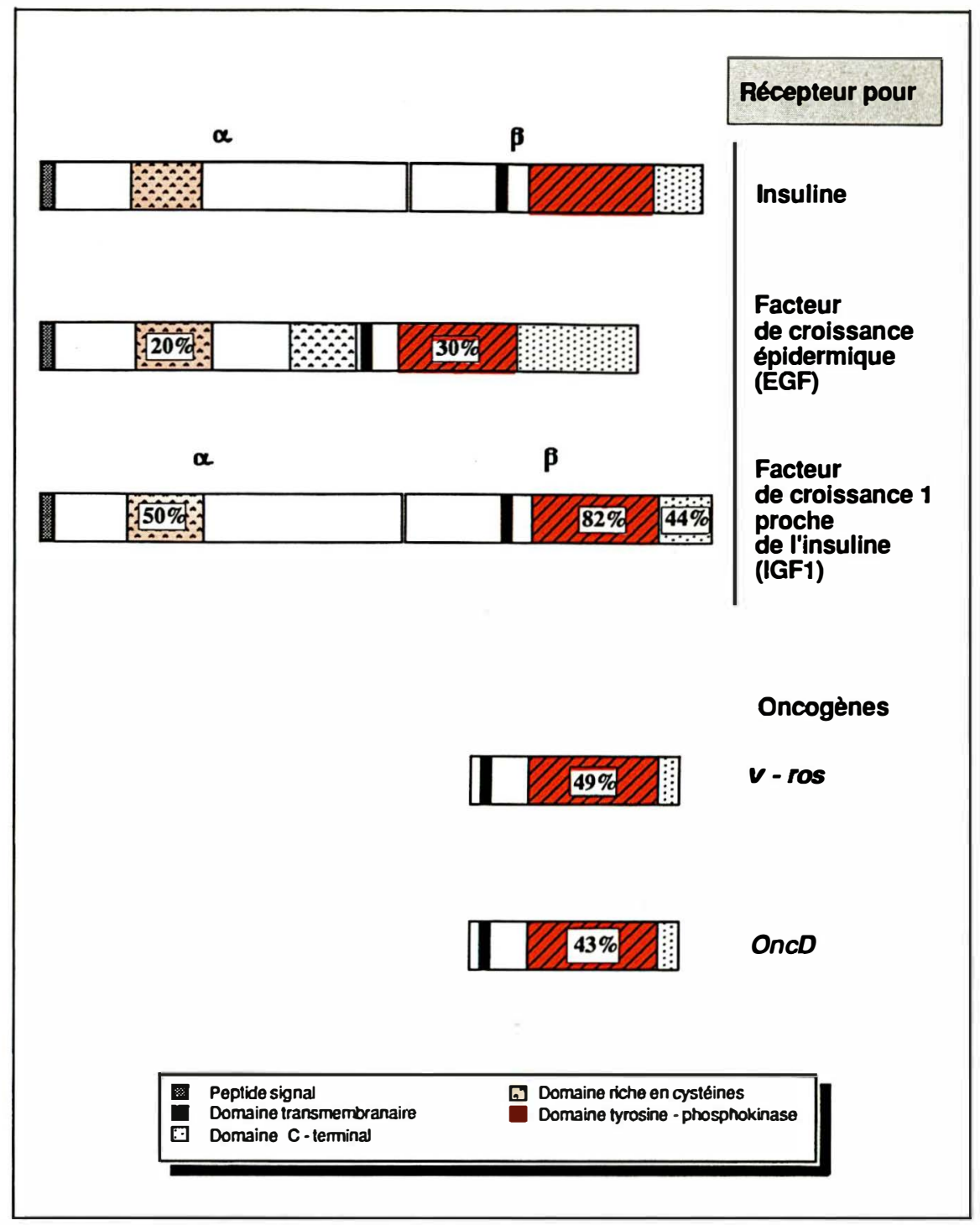

Figure 2. Comparaison structurale des récepteurs de I'EGF, de I'IGFI et des oncogènes v-ros et oncD au récepteur de l'insuline. Les homologies supérieures ou égales à $20 \%$ sont notées dans les domaines correspondants.

structure peu spécifiques mais nécessaires à la transduction du signal hormonal. L'extrapolation de ces résultats et de leur interprétation au ligand spécifique que représente l'insuline, ont conduit à imaginer que l'insuline agissait en modifiant la structure d'un' récepteur tétramérique $\alpha_{2} \beta_{2}$.

\footnotetext{
* $K_{D}$ : constante de dissociation jugeant de l'affinité d'un ligand $(H)$ pour son récepteur (R) $\left(H R \stackrel{K_{D}}{\rightleftarrows} H+R\right)$.
}

Cependant, des études in vitro montrent qu'un récepteur $\alpha \beta$ lie normalement l'insuline et qu'il détermine l'activation de la tyrosine kinase de la chaine $\beta$ [9]. La réalité physiologique d'un récepteur $\alpha \beta$ ne peut donc pas être totalement écartée.

\section{Domaines fonctionnels}

Fonction de liaison de l'insuline. La liaison de l'insuline à son récepteur a été particulièrement étudiée. Cette liaison est caractérisée par sa haute affinité $\left(\mathrm{K}_{\mathrm{D}} \simeq\right.$ $\operatorname{lnM})^{*}$ et par sa spécificité mar- 


\section{RÉFÉRENCES}

11. Pang DT, Shafer JA. Evidence that insulin receptor from human placenta has a high affinity for only one molecule of insulin. $J$ Biol Chem 1984; 259 : 8589-96.

12. Davis CG, Goldstein JL, Sudhof TC, Anderson RGW, Russel DW, Brown MS. Acid-dependent ligand dissociation and recycling of LDL receptor mediated by growth factor homology region. Nature 1987; 326 : 760-5.

13. Kahn CR. The molecular mechanism of insulin action. Annu Rev Med 1985 ; 36 : 42951

14. Czech MP. The nature and regulation of the insulin receptor: structure and function. Ann Rev Physiol 1985 ; 47 : 357-81.

15. Gammeltoft S, Van Obberghen E. Protein kinase activity of the insulin receptor. Biochem J 1986; 235 : 1-11.

16. Rosen OM. After insulin binds. Science $1987 ; 237: 1452-8$.

17. Tornquist HE, Pierce MW, Frackelton $R$, Nemenoff RA, Avruch J. Identification of insulin receptor tyrosin residues autophosphorylated in vitro. J Biol Chem 1987; 262 : 10212-9.

18. Ellis L, Clauser E, Morgan D, Edery M, Roth RA, Rutter WJ. Replacement of insulin receptor tyrosine residues 1162 and 1163 compromises insulin-stimulated kinase activity and uptake of 2-deoxyglucose. Cell 1986; 45: 721-32.

19. Cohen SH, Ushiro H, Stoscheck C, Chinkers M. A native 170000 epidermal growth factor receptor-kinase complex from shed plasmid membrane vesicles. J Biol Chem 1982 ; 257 : 1523-31.

20. Chou CK, Dull T J, Russell DS, et al. Human insulin receptors mutated at the ATP-binding site lack protein tyrosine kinase activity and fail to mediate postreceptor effects of insulin. J Biol Chem 1987; 262 : quée. Le ou les site(s) moléculaire(s) d'interaction ont bien été déterminés pour l'insuline [10] mais restent inconnus pour son récepteur. Les analyses et les comparaisons structurales du récepteur de l'insuline et de peptides apparentés suggèrent cependant que le site de liaison doit être localisé dans les régions situées de part et d'autre du domaine riche en cystéines de la chaîne $\alpha$, car ces régions sont moins bien conservées que le domaine riche en cystéines qui pourrait, quant à lui, présenter une structure compacte formée d'un réseau de ponts disulfures. Malgré son implication dans la liaison des LDL à leur récepteur, cette dernière région est un mauvais candidat pour le site de liaison lui-même, en raison de son fort potentiel hydrophile et de sa relative conservation à travers la famille de récepteurs.

Le nombre de molécules d'insuline se liant à une unité monomérique $\alpha \beta$ est encore l'objet de controverses. La plupart des études s'accordent pour montrer qu'une seule molécule d'insuline se lie à une chaîne $\alpha[10]$. Cependant, un travail récent montrait que chaque unité du récepteur de l'insuline liait deux molécules d'insuline, mais que les deux sites de liaison avaient une affinité très différente pour l'insuline[11]. Ceci est à rapprocher des observations de P. de Meyts [10] qui a montré que l'aff inité du récepteur de l'insuline pour son ligand diminuait avec l'augmentation de l'occupation des sites. Ce phénomène, appellé coopérativité négative, pourrait expliquer l'aspect curvilinéaire des courbes de liaison exprimées selon Scatchard ${ }^{*}$ et pourrait faire partie d'un phéno-

\footnotetext{
- Courbe selon Scatchard: représentation graphique de la liaison d'un ligand pour son récepteur, exprimant en abscisse le rapport du ligand lié sur le ligand libre et en ordonnée la concentration de ligand lié. Une représentation linéaire signifie qu'il n'y a qu'un seul type de sites de liaison. La pente de cette droite indique le $K_{D}$ et l'intersection de la droite avec la ligne des abscisses représente le nombre de sites.
}

mène plus général correspondant à des modifications de structure de la sous-unité $\alpha$, observées très rapidement après la liaison de l'insuline et modifiant les caractéristiques physicochimiques du récepteur

Le devenir du récepteur après liaison de l'insuline à son domaine extracellulaire a également été bien étudié et fait l'objet d'un article publié dans ce numéro de médecine/science. Cependant les bases moléculaires de ces phénomènes d'agrégation et d'endocytose sont encore inconnues. Un travail très récent sur le récepteur des LDL [12] a montré qu'une séquence de 40 acides aminés dans le domaine riche en cystéines était conservée dans les séquences d'autres récepteurs et protéines membranaires, tel le récepteur de l'EGF. La délétion de cette séquence ne modifiait pas la liaison des LDL, mais après internalisation, ce récepteur modifié ne pouvait ni se débarrasser de son ligand ni être recyclé. Il était rapidement dégradé. La démonstration que des séquences homologues du récepteur de l'insuline pourraient jouer un tel rôle, reste a apporter.

Fonction tyrosine-kinase et autophosphorylation [13-16]. La liaison de l'insuline active l'autophosphorylation et la fonction tyrosine-kinase de la chaîne $\beta$. La phosphorylation de la chaîne $\beta$ est la modification la plus précoce observée après liaison de l'insuline à la chaîne $\alpha$. Cette phosphorylation porte sur des résidus tyrosyls qui ont récemment pu être identifiés. En effet, in vitro, les tyrosines 1158,1162 et 1163 du domaine tyrosine kinase et les tyrosines 1328 et 1334 du domaine C terminal sont phosphorylées [17]. Parallèlement, le récepteur acquiert une activité enzymatique de type tyrosine kinase après avoir lié l'insuline. Les caractéristiques biochimiques de cette activité enzymatique ont été bien établies : l'activation de l'enzyme apparaît quelques secondes après la liaison du ligand, la source de phosphate est l'ATP et l'activité enzymatique est poten- 


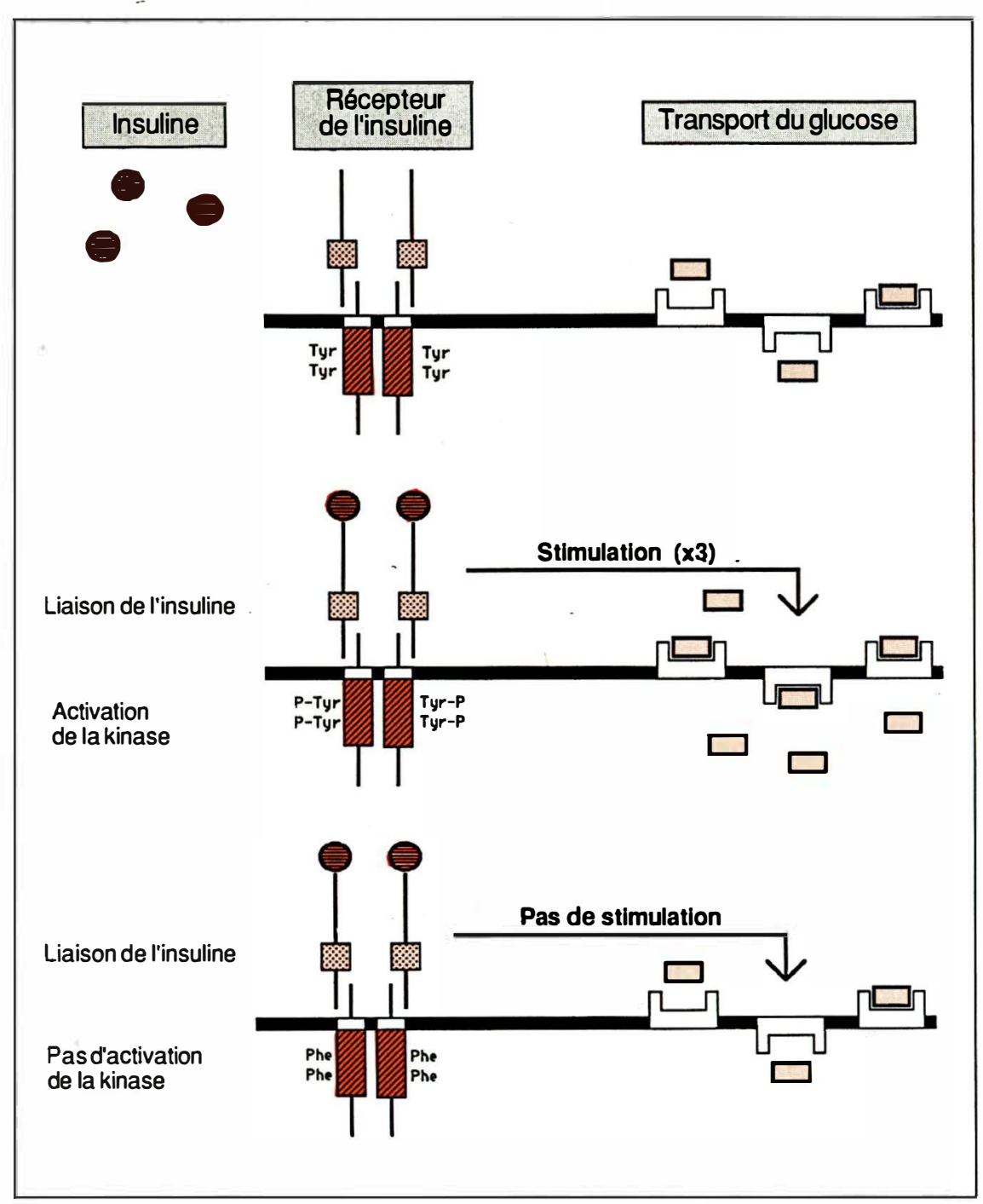

Figure 3. Actions de l'insuline sur le transport du glucose par l'intermédiaire de son récepteur. En haut, en l'absence d'insuline liée, le récepteur n'exerce aucun effet sur le transport basal du glucose. Au milieu la fixation de l'insuline à son récepteur détermine une phosphorylation du récepteur, l'activation de sa tyrosine kinase et la stimulation du transport du glucose. En bas, la mutation du site majeur d'autophosphorylation du récepteur (tyr 1162-tyr 1163 en phe) supprime l'activation de la kinase et la stimulation du transport du glucose. L'insuline est schématisée sous la forme d'un cercle hachuré horizontalement. Le récepteur est schématisé sous la forme d'un hétérodimère dont les domaines riches en cystéines (pointillé) et tyrosine kinases (hachuré transversal) sont représentés par des rectangles.

tialisée par des ions divalents tels que le $\mathrm{Mg}^{++}$et le $\mathbf{M n}^{++}$. La modification enzymatique consécutive à la liaison du ligand porte essentiellement sur la vitesse de la réaction (Vmax augmentée 5 à 20 fois) et non sur le $\mathrm{Km}^{*}$, c'est-

\footnotetext{
- Km: constante de Michaelis-Menten. $m / s n^{\circ} 2$ vol. 4, février 88
}

tyrosine kinase paraît évidente. Ainsi, la perte du site d'autophosphorylation, que représente la mutation des tyrosines 1162-1163 en phénylalanines, conduit l'insuline à ne pas pouvoir activer la kinase d'un tel récepteur [18].

Parallèlement, le récepteur de l'EGF a fait aussi l'objet de nombreux travaux, qu'il est intéressant de comparer à ceux portant sur le récepteur de l'insuline. Le récepteur de l'EGF possède trois sites d'autophosphorylation dans le domaine $\mathrm{C}$ terminal de la portion intracellulaire. La délétion de ce domaine conduit à la réduction des possibilités d'autophosphorylation du récepteur lors de la liaison de l'EGF, mais est sans influence sur la capacité d'activation de la tyrosine kinase et sur la transmission du signal [19]. Une telle dissociation fonctionnelle entre l'autophosphorylation et l'activité tyrosine kinase est également observée pour certaines des protéines transformantes codées par les oncogènes tels pp60src. Ainsi, les sites d'autophosphorylation du récepteur de l'EGF et du domaine $\mathbf{C}$ terminal du récepteur de l'insuline ne semblent pas influencer l'activation de la tyrosine kinase et leur phosphorylation pourrait être une conséquence de l'activation de la kinase. A l'opposé, le site d'autophosphorylation représenté par les tyrosines 1162-1163 semble jouer un rôle actif, bien qu'obscur, dans l'activation de la tyrosine kinase. Deux classes de tyrosine kinases peuvent ainsi être définies selon que l'autophosphorylation joue un rôle ou non dans l'activation de l'enzyme.

L'activation de la kinase est responsable des actions de l'insuline. La connaissance de la séquence primaire et la possession des ADNc codant pour ces récepteurs ont permis des progrès rapides, bien qu'incomplets, dans la compréhension du rôle de la tyrosine kinase dans la transmission du signal. La première démonstration directe d'un lien étroit entre activation de la kinase et l'action cellulaire de l'insuline, a pu être 


\section{RÉFÉRENCES}

21. Morgan DO, Roth RA. Acute insulin action requires insulin receptor kinase activity : introduction of an inhibitory monoclonal antibody into mammalian cells blocks the rapid effects of insulin. Proc Natl Acad Sci USA 1987 ; 84 : 41-5.

22. Ellis L, Morgan DE, Koshland DE Jr, et al. Linking functionnal domains of the human insulin receptor with the bacterial aspartate receptor. Proc Natl Acad Sci USA $1986 ; 83: 8137-41$.

23. Ellis L, MorgandO, JongSM, Wang LH, Roth RA, Rutter WJ. Heterologous transmembrane signaling by a human insulin receptor-v-ros hybrid in Chinese hamster ovary cells. Proc Natl Acad Sci USA $1987 ; 84: 5101-5$.

24. Riedel H, Dull T J, Schlessinger J, Ullrich A. A chimaeric receptor allows insulin to stimulate tyrosine kinase activity of epidermal growth factor receptor. Nature $1986 ; 324$ : 68-70.

25. Ellis L, Morgan D, Clauser E, Roth RA, Rutter WJ. A membrane-anchored cytoplasmic domain of the human insulin receptor mediates a constitutively elevated insulinindependent uptake of 2-deoxyglucose. Mol Endocrinol 1987 ; 1 : 15-24.

26. Colca JR, Dewald DB, Pearson JD, Palazuk BJ, Laurino JP, McDonald JM. Insulin stimulates the phosphorylation of calmodulin in intact adipocytes. J Biol Chem 1987; 262 : 11399-402.

27. Saltiel AR, Fox JA, Sherline P, Cuatrecasas P. Insulin-stimulated hydrolysis of a novel glycolipid generates modulators of cAMP faite. En effet la mutation des tyrosines 1162 et 1163 (citée plus haut et que nous avons définies comme sites potentiels d'autophosphorylation du récepteur de l'insuline) en phénylalanines a été effectuée de façon spécifique par mutagenèse dirigée de l'ADNc. L'expression de cet ADN muté codant pour ce récepteur dans une lignée cellulaire eucaryote $\mathrm{CHO}$ (lignée établie dérivée d'une tumeur ovarienne de hamster chinois) démontre que ce récepteur lie normalement l'insuline; cependant, contrairement à ce qui est observé pour le récepteur «sauvage», cette liaison ne stimule pas ou peu l'autophosphorylation et l'activité tyrosine kinase du récepteur. En corollaire, le transport du glucose à travers la membrane plasmique ne peut plus être stimulé par l'insuline (figure 3 , page 77) [18].

Cette première étude a depuis été confirmée et étendue à d'autres actions de l'insuline et d'autres types cellulaires. Ainsi l'incapacité de l'insuline à stimuler la kinase d'un récepteur dont le site de liaison de l'ATP a été muté s'accompagne de l'absence d'effet de l'hormone sur le transport du glucose, la synthèse de glycogène, la synthèse d'ADN, l'activation de différentes phosphorylations cellulaires et l'activation de la kinase S6 [20]. De même, des anticorps dirigés contre le site d'autophosphorylation (tyrosines 1162-1163) bloquent plusieurs effets de l'insuline comme la captation du glucose, l'incorporation de thymidine tritiée, la synthèse de glycogène [21] dans différents tissus (foie, tissu adipeux, fibroblastes), ainsi que la maturation de l'œuf de grenouille. Ces premiers résultats doivent cependant être confirmés avant d'affirmer que la totalité des effets insuliniques passent par l'activation de la tyrosine kinase du récepteur. En effet, certaines observations isolées d'effets insulino-mimétiques d'anticorps polyclonaux anti-récepteurs, sans activation de la kinase, doivent rendre prudent.

Les rôles respectifs du domaine de liaison de l'hormone et du domaine intracellulaire à activité tyrosine kinase ont été analysés grâce à la synthèse, obtenue par recombinaison génétique, de protéines chimériques associant des domaines de différents récepteurs. Ainsi l'expression d'un récepteur comportant la chaîne $\alpha$ du récepteur de l'insuline et le domaine intracellulaire enzymatique du récepteur bactérien à l'aspartate, a $\mathrm{pu}$ être réalisée dans la lignée $\mathrm{CHO}$ [22]. Ce récepteur lie normalement l'insuline, mais n'est pas fonctionnel. Plus intéressants sont les remplacements du domaine intracellulaire $d u$ récepteur de l'insuline par le domaine homologue de v-ros [23] ou du récepteur de l'EGF [24]. Dans les deux cas, l'autophosphorylation et la tyrosine kinase du récepteur chimérique sont activées par l'insuline sans induction des modifications métaboliques normalement provoquées par l'hormone. Le mécanisme par lequel le domaine extracellulaire transmet le signal au domaine intracellulaire n'est pas connu. Cependant, l'expression de formes délétées du récepteur de l'insuline a permis de montrer que la suppression du domaine de liaison du ligand conduisait à l'établissement d' une fonction constitutive mimant la stimulation continue par le ligand [25]. Ainsi, une élévation permanente du taux de captation du glucose a pu être observée dans une lignée cellulaire $\mathrm{CHO}$ exprimant une forme membranaire de la chaîne $\beta$ du récepteur (figure 4). Cet effet n'était pas observé lorsque cette même chaîne $\beta$ était mutée au niveau de ses tyrosines jumelles ou était exprimée sous une forme cytosolique monomérique. Ces faits sont en faveur de l'hypothèse selon laquelle la chaîne $\alpha$ exercerait, au repos, un effet inhibiteur sur la chaîne $\beta$, effet qui serait levé par la liaison du ligand ou la suppression de la chaîne $\alpha$.

Il semble donc que la plupart des actions de l'insuline passent par l'activation de la tyrosine kinase, mais les étapes suivant cette activation restent inconnues. In vitro, les substrats de cette tyrosine 
kinase sont nombreux, mais leur spécificité in vivo est discutable. Deux protéines de poids moléculaires de $180 \mathrm{kDa}^{*}$ et $15 \mathrm{kDa}$ respectivement ont été identifiées récemment et semblent de bons candidats. La calmoduline a été identifiée comme la protéine de $15 \mathrm{kD}$ [26], ce qui ouvre des horizons nouveaux. Ceci ne représente cependant qu'un possible maillon d'une chaîne qui est encore loin d'être analysée. En effet, si on peut concevoir aisément que la tyrosine kinase du récepteur puisse intervenir dans l'activation ou l'inhibition de certaines voies métaboliques par des cascades de phosphorylations impliquant certaines sérine kinases activées par phosphorylation de résidus tyrosyls, l'intervention de ces phosphorylations reste plus difficile à comprendre pour certains effets de l'insuline (l'activation de la captation du glucose ne passe pas par la phosphorylation du transporteur et on imagine encore mal comment la synthèse des protéines peut être activée par phosphorylation).

Domaine C-terminal et second messager. Le développement récent des connaissances sur le rôle de la tyrosine kinase du récepteur peut sembler rendre caduque l'hypothèse selon laquelle un peptide détaché du récepteur lors de la liaison de l'insuline pourrait jouer le rôle de second messager. Un tel médiateur de nature peptidique avait été identifié il y a quelques années à partir d'extraits membranaires stimulés par l'insuline. La nature précise de ces messagers n'a cependant pas été confirmée. L'analyse structurale du récepteur de l'insuline après clonage a donné un regain d'intérêt à cette théorie, en révélant l'existence d'une région $\mathrm{C}$ terminale spécifique du récepteur, très hydrophile et mobile dans le cytoplasme, pouvant théoriquement libérer deux peptides par clivage trypsique de résidus dibasiques. L'ex-

\footnotetext{
* kDa: kilodalton.

$\mathrm{m} / \mathrm{s} n^{\circ} 2$ vol. 4, fevrier 88
}

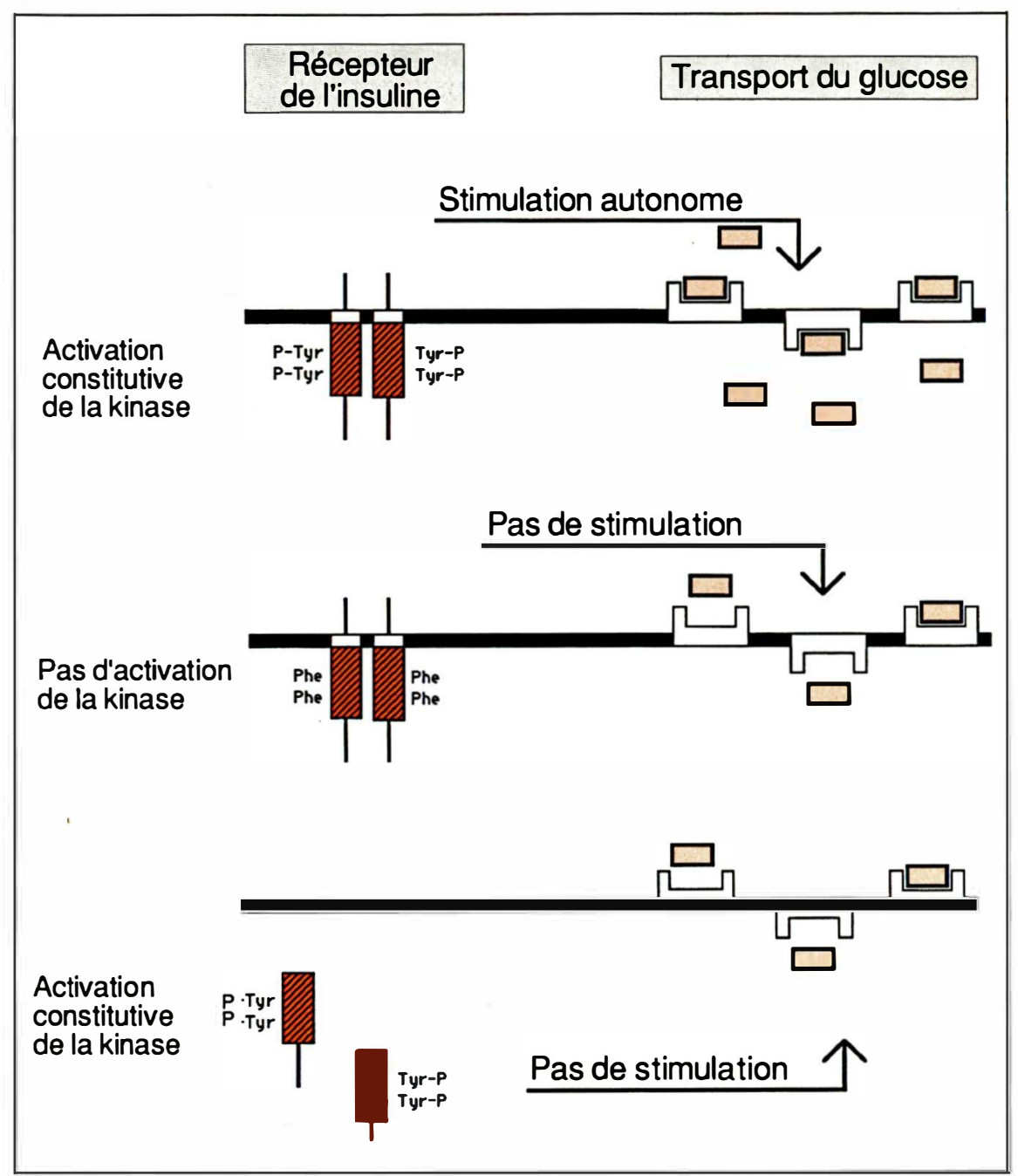

Figure 4. Activité de formes tronquées du récepteur de l'insuline sur la captation de glucose. Dans la partie supérieure du schéma, une forme membranaire de la chaîne $\beta$ seule voit sa tyrosine kinase activée de façon constitutive et détermine une stimulation du transport du glucose, permanente et indépendante de l'insuline. Cet effet n'est pas observé si le site d'autophosphorylation est muté (partio médiane) ou si la tyrosine kinase activée est produite dans le cytoplasme (partio inférieure).

pression d'un récepteur, ne comportant pas de région $\mathrm{C}$ terminale, n'a pas permis de parvenir à une conclusion définitive sur ce point, la suppression de cette région entraînant une complète destructuration tridimensionnelle de la chaîne $\beta$ [18]. L'étude de récepteurs de l'insuline ayant des délétions C-terminales plus limitées, ainsi que les peptides synthétiques correspondants sont actuellement étudiés; les premiers résultats ne sont pas en faveur du rôle physiologique de ces peptides. D'autre part, l'existence de médiateurs de faible poids moléculaire de nature lipidique ou glucidique a été suspectée. Ainsi, un médiateur de nature glucido-lipidique, libéré sous l'effet de l'insuline, d'un précurseur de type phosphatidyl-inositolglycane a récemment été isolé [27]. Ce glycolipide module l'action de la phosphodiestérase AMPc dépendante, mais aussi d'autres enzymes comme la pyruvate déshydrogénase et l'adénylate cyclase, et simule les effets lipolytiques de l'insuline. Tout récemment, ce second messager s'est révélé mimer l'ensemble des actions de phosphorylation et de 
déphosphorylation de l'insuline dans des adipocytes [28]. Il est cependant encore difficile de faire la connexion entre ce médiateur et la tyrosine kinase du récepteur.

\section{Vers une pathologie génétique}

Le gène du récepteur de l'insuline est localisé au niveau du chromosome 19. Bien que sa structure moléculaire ne soit pas connue, il semblerait que sa taille, appréciée par Southern blot, soit supérieure a $100 \mathrm{~kb}$ (kilobases) [2]. La disponibilité des sondes d'ADNc a conduit à la multiplication des études à la recherche de polymorphismes de la longueur des fragments de restriction (RFLP) du gène du récepteur de l'insuline. Ainsi des polymorphismes $R s a l$, Puull et Stul [29] ont-ils été décrits dans une population normale avec des fréquences variables.

Plus intéressantes étaient les recherches de modifications majeures de la structure du gène associées à d'éventuelles anomalies du récepteur. En première intention, celles-ci ont été recherchées parmi les maladies et syndromes associés à une insulinorésistance. Les insulino-résistances peuvent être classées sur un plan physiopathologique en insulino-résistances de niveaux, «pré-récepteurs», « récepteurs » et «post-récepteurs», que ces insulino-résistances soient congénitales ou acquises [30, 31] (1) Les insulinorésistances «prérécepteur » sont essentiellement liées à des anomalies de l'insuline (hyperproinsulinémies familiales, insulines mutantes) ou à la production d'anticorps anti-insuline. (2) Les insulinorésistances de niveau « récepteur» sont définies par une anomalie de liaison de l'insuline exogène au niveau du récepteur. Ceci regroupe les anomalies liées aux anticorps antirécepteurs circulants observées dans l'insulino-résistance, avec Acanthosis nigricans de type $\mathrm{B}$ ou en association avec certains déficits immunologiques (ataxietélangiectasie, déficit en IgA) [32],

les diminutions acquises du nom- bre des récepteurs telles que celles observées dans le diabète de type II et l'obésité et enfin certains syndromes cliniques très rares comme l'insulino-résistance avec Acanthosis nigricans de type A, le diabète lipoatrophique et le lepréchaunisme. Ces syndromes, dont les caractéristiques cliniques sont résumées sur le Tableau I, s'accompagnent tous d'une insulinorésistance avec dans certains cas une anomalie qualitative ou quantitative du récepteur. (3) Les insulinorésistances «post-récepteurs » regroupent toutes les insulino-résistances qui ne sont pas associées à une anomalie de l'insuline ou de sa liaison au récepteur. Ce groupe inclut donc, sur un plan physiopathologique, toutes les anomalies de transduction intracellulaire du signal porté par l'insuline; il peut toucher la tyrosine-kinase du récepteur ou les voies effectrices. Classiquement, en font partie les insulino-résistances associées aux principaux désordres endocriniens par hypersécrétion d'une hormone de contre-régulation (hormone de croissance dans l'acromégalie, ACTH dans le syndrome de Cushing, glucagon dans le glucagonome, catécholamines dans le phéochromocytome) ou encore le diabète non insulino-dépendant et l'obésité. Enfin l'insulino-résistance avec Acanthosis nigricans de type C, le diabète lipoatrophique et le lepréchaunisme ne comportent pas toujours une anomalie décelable du récepteur.

Ainsi les syndromes qui sont les candidats les plus susceptibles d'être associés à une anomalie génétique de la structure du récepteur de l'insuline sont-ils l'insulino-résistance avec Acanthosis nigricans de type $\mathrm{A}$, le diabète lipoatrophique et le léprechaunisme. Ces trois maladies rares, dont les signes cliniques sont différents, sont réunies par leur caractère congénital possible et l'insulino-résistance majeure qui domine le tableau biologique (Tableau I). Elles ont fait l'objet d'études récentes afin d'identifier une éventuelle anomalie du 79 : 1330-7. 
récepteur de l'insuline. Ainsi l'insulino-résistance avec Acanthosis nigricans de type A semble parfois liée à une anomalie de biosynthèse ou de maturation du récepteur. Certains diabètes lipoatrophiques sont associés à une anomalie de phosphorylation des récepteurs de l'insuline et de l'IGFl sans anomalie de liaison de l'insuline. Enfin une anomalie biochimique du récepteur de l'insuline (sensibilité anormale au $\mathrm{pH}$ et à la température) a été identifiée dans un cas de lepréchaunisme [33].

L'étude de la structure du gène du récepteur de l'insuline de ces patients a débuté grâce à la technique de Southern blot. Elle est rendue difficile par la rareté de ces syndromes. L'étude d'une famille, dont un enfant présentait un tableau clinique d'insulino-résistance de type $\mathrm{A}$, a révélé une modification majeure de la structure du gène du récepteur de l'insuline, dont la séquence nucléotidique est en cours d'analyse. Nous avons, pour notre part, intensivement étudié le gène du récepteur de l'insuline de six patients ayant un diabète lipoatrophique sans indice d'une liaison avec la maladie à ce jour. Ceci peut être dû soit au caractère grossier de la méthode d'analyse, qui ne peut détecter des mutations limitées, soit au fait que la maladie n'est en fait pas liée à une anomalie du gène du récepteur de l'insuline.

Des altérations du récepteur de l'insuline sont également recher- chées dans le diabète non insulino-dépendant de type II. De nombreuses études, en particulier sur les jumeaux, ont en effet montré que des facteurs génétiques contribuaient à la susceptibilité à ce type de diabète, dont une des caractéristiques est l'existence d'un état d'insulino-résis tance [34], ce qui suggère qu'une anomalie de fonction du récepteur pourrait, dans certains cas au moins, participer à l'état pathologique. De fait, de plus en plus d'études démontrent l'apparition d'anomalies de fonctionnement de la tyrosine kinase du récepteur, aussi bien dans les modèles animaux d'obésité et de diabète de type II que chez l'homme [35]. Le gène du récepteur de l'insuline dans le diabète de type II a fait

\begin{tabular}{|c|c|c|c|}
\hline \multicolumn{4}{|c|}{$\begin{array}{l}\text { SYNDROMES D'INSULINO-RÉSISTANCE MAJEURE } \\
\text { ANOMALIES GÉNÉTIQUES POSSIBLES DU RÉCEPTEUR DE L'INSULINE }\end{array}$} \\
\hline & $\begin{array}{l}\text { Insulinorésistance } \\
\text { de type A }\end{array}$ & Diabète lipoatrophique & Lepréchaunisme \\
\hline $\begin{array}{l}\text { Clinique } \\
\text { Découverte }\end{array}$ & Adolescence & Variable & Naissance \\
\hline Développement & $\begin{array}{l}\text { Avance staturo-pondérale } \\
\text { Acanthosis nigricans }{ }^{*}\end{array}$ & $\begin{array}{l}\text { Avance staturo-pondérale } \\
\text { Acanthosis nigricans* }\end{array}$ & Retard staturo-pondéral \\
\hline Signes cliniques & Hyperandrogénie & $\begin{array}{l}\text { Hyperandrogénie } \\
\text { Lipoatrophie } \\
\text { Hypertrophie musculaire } \\
\text { Hépatomégalie }\end{array}$ & $\begin{array}{l}\text { Atrophie sous-cutanée } \\
\text { Dysmorphie }\end{array}$ \\
\hline Biologie & $\begin{array}{l}\text { Hyperglycémie } \\
\text { Hyperinsulinémie }\end{array}$ & $\begin{array}{l}\text { Hyperglycémie } \\
\text { Hyperinsulinémie } \\
\text { Hypertriglycéridémie }\end{array}$ & Hyperinsulinémie \\
\hline $\begin{array}{l}\text { Anomalies du récepteur } \\
\text { Structure }\end{array}$ & - & - & 1 cas \\
\hline Liaison & Normale ou diminuée & Normale & Diminuée en général \\
\hline Tyrosine kinase & Normale & Diminuée & Normale en général \\
\hline $\begin{array}{l}\text { Gène du récepteur } \\
\text { anormal }\end{array}$ & 1 cas & Non & 1 cas \\
\hline
\end{tabular}

* Acanthosis nigricans : dermatose rare traduite par des placards d'hyperkératose hyperpigmentée. 
l'objet de deux courtes études contradictoires : chez des sujets de race blanche, $78 \%$ des diabétiques présentaient deux bandes supplémentaires après digestion par l'enzyme de restriction Bgl2, alors que $41 \%$ des témoins présentaient cette caractéristique. Une étude japonaise sur 26 diabétiques et 19 témoins utilisant trois enzymes différentes n'a pas montré de différences [29].

Devant le caractère très préliminaire et fragmentaire des résultats et les difficultés d'analyse, il paraît donc impossible de conclure aujourd'hui sur la réalité d'anomalies génétiques du récepteur de l'insuline responsables de syndromes pathologiques, comme c'est le cas par exemple pour le récepteur des LDL.

En conclusion. La structure primaire du récepteur de l'insuline est bien établie et a contribué à la connaissance des domaines fonctionnels de cette molécule. L'étude cristallographique de chacune des deux chaînes de la protéine, produites par expression de l'ADN recombinant, devrait apporter le complément d'informations indispensable à la définition d'un site de liaison de l'insuline, modèle logique pour la synthèse d'analogues de l'insuline, et à la meilleure connaissance du fonctionnement enzymatique de la tyrosine kinase.

$\mathrm{La}$ «dissection » moléculaire du récepteur de l'insuline actuellement en cours par mutagenèse dirigée et analyse fonctionnelle des mutants devrait permettre de préciser certains aspects du fonctionnement du récepteur (interactions entre les chaînes $\alpha$ et $\beta$, relations entre autophosphorylation et activation de la tyrosine kinase, rôle des chaînes glycosidiques, etc.).

Enfin une analyse approfondie de la structure biochimique et génétique du récepteur de l'insuline dans le groupe hétérogène des diabétiques non insulino-dépendants, devrait permettre d'identifier un syndrome d'insulino-résistance consécutif à une anomalie qualitative ou quantitative du récepteur de l'insuline

\section{Summary}

The insulin receptor is a membrane-bound glycoprotein formed by two extracellular alpha chains, involved in insulin binding and two beta chains, exhibiting a tyrosine-kinase activity. The association of ligand binding and enzymatic activity in the same molecule defines a new class of receptor, including epidermal growth factor (EGF) receptor, insulin like growth factor I (IGFI) receptor and other growth factor receptors. The recent cloning of the cDNA coding for the human insulin receptor allowed us to deduce the primary structure of this receptor and to identify some functionnal domains. Thus the structure-function relationships of the insulin receptor were explored using the powerful tools represented by the recombinant DNA and the immunological technologies. Receptors, modified by site-directed mutagenesis or deletions, were expressed in eucaryotic cells and their functions were tested. The actions of antibodies directed against different epitopes of the insulin receptor, were also investigated. These experiments demonstrate the crucial role of the tyrosinekinase activation in mediating the insulin actions. Moreover the identification of clinical cases of insulino-resistance, associated with abnormalities of the insulin receptor structure or function and gene polymorphism, has started.

\section{TIRÉS A PART}

E. Clauser : Inserm U. 36, 17, rue du Fer à Moulin, 75005 Paris, France. 